\title{
Karyotype relationships among Anastrepha bistrigata, $A$. striata and $A$. serpentina (Diptera, Tephritidae)
}

\author{
Denise Selivon ${ }^{1}$, Fabiana Maria Sipula ${ }^{1}$, Lincoln S. Rocha ${ }^{1,2}$ and André Luiz P. Perondini ${ }^{1}$ \\ ${ }^{1}$ Departamento de Genética e Biologia Evolutiva, Instituto de Biociências, Universidade de São Paulo, \\ São Paulo, SP, Brazil. \\ ${ }^{2}$ Laboratório de Parasitologia, Instituto Butantan, São Paulo, SP, Brazil.
}

\begin{abstract}
The species of Anastrepha are arranged into 17 intrageneric groups. Recently, it was proposed that two species of the striata group, Anastrepha striata and $A$. bistrigata, might be realocated to serpentina group. Anastrepha bistrigata and $A$. serpentina have an $\mathrm{X}_{1} \mathrm{X}_{2} \mathrm{Y} / \mathrm{X}_{1} \mathrm{X}_{1} \mathrm{X}_{2} \mathrm{X}_{2}$ sex chromosome system while $A$. striata has a $\mathrm{XY} / \mathrm{XX}$ system. It was previously proposed that the karyotype of $A$. bistrigata could be derived from that of $A$. striata by an Y:A fusion, and that the karyotype of $A$. serpentina would be derived from another, hypothetical karyotype. In the present report sequential staining with DAPI and chromomycin $\mathrm{A} 3\left(\mathrm{CMA}_{3}\right)$, followed by C-banding, revealed that the C-banded heterochromatic blocks of the sex chromosomes of $A$. bistrigata have different affinities to fluorochromes in comparison to the chromosomes of $A$. striata, from which they have hypothetically derived. The chromosomes of $A$. serpentina show substantial differences in their cytochemical properties compared to their $A$. bistrigata and $A$. striata counterparts. The FISH technique showed that the ribosomal gene sequences are located in DAPI- or DAPI/CMA ${ }_{3}$-positive heterochromatic blocks of the sex chromosomes, one site on the $\mathrm{Y}$ chromosome and one site on the $\mathrm{X}$ chromosome ( $\mathrm{X}_{1}$ in $A$. bistrigata and $A$. serpentina). The data suggest that the karyotype of $A$. striata and $A$. bistrigata could be derived from a common ancestral karyotype, while the $A$. serpentina karyotype probably has a distinct origin.
\end{abstract}

Key words: fruit flies, mitotic chromosomes, fluorochromes, heterochromatin.

Received: June 14, 2007; Accepted: August 22, 2007.

\section{Introduction}

Anastrepha is a genus of frugivorous fruit flies endemic to the American continent and it encompasses about 200 described species (White and Elson-Harris, 1992; Norrbom et al., 1999; Zucchi, 2000). Although the biology of Anastrepha has been the object of several studies, cytogenetic analyses are scarce: only 23 species had their mitotic chromosomes described (Selivon et al., 2005a, 2005b). The first reports described only the number, morphology and types of sex chromosome systems (Metz, 1916; Mendes, 1958; Bush, 1962). Later, the size and distribution of heterochromatic blocks were also included in the descriptions (Solferini and Morgante, 1987,1990; Morgante et al., 1993, 1996; Basso and Manso, 1998; Basso et al., 2003; Selivon et al., 2004, 2005a, 2005b). More recently, the cytochemical properties of the heterochromatin as well as the location of ribosomal sequences were investigated in eight Anastrepha species (Goday et al., 2006), pro-

Send correspondence to Denise Selivon. Departamento de Genética e Biologia Evolutiva, Instituto de Biociências, Universidade de São Paulo, Rua do Matão 277, 05508-900 São Paulo, SP, Brazil. E-mail: dselivon@ib.usp.br. viding new tools for the cytotaxonomy of this group of flies.

The Anastrepha species are arranged into 17 intrageneric taxonomic groups based on morphological traits (Norrbom et al., 1999) and on phylogenetic analyses using mitochondrial (McPheron et al., 1999) or nuclear genes (Barr et al., 2005). Anastrepha striata and A. bistrigata are sister taxa which were assigned to the striata group, but a recent taxonomic revision proposed the realocation of both to the serpentina group (Norrbom, 2002). Anastrepha striata has a $2 \mathrm{n}=12$, and an XX/XY sex chromosome system, while $A$. bistrigata and $A$. serpentina show a neo$\mathrm{Y} /$ neo-X sex chromosome system $\left(\mathrm{X}_{1} \mathrm{X}_{2} \mathrm{Y} / \mathrm{X}_{1} \mathrm{X}_{1} \mathrm{X}_{2} \mathrm{X}_{2}\right.$, $2 n=11$ in males, $2 n=12$ in females) (Bush, 1962; Solferini and Morgante, 1987). In another report, Solferini and Morgante (1990) proposed that the karyotype of A. bistrigata may have derived from $A$. striata through fusion of the $\mathrm{Y}$ to an acrocentric autosome, giving origin to the neo-Y/neo-X chromosomes. They also proposed that the karyotypes of $A$. serpentina could be derived from a hypothetical karyotype (with XX/XY sex chromosomes) through a Y:A fusion. 
In the present study, mitotic chromosomes of $A$. striata and $A$. bistrigata were sequentially stained with the fluorochromes DAPI and chromomycin A3 that bind, respectively, to AT- and GC-rich chromosome regions (Schweizer, 1981), followed by C-banding. The chromosomes of $A$. serpentina have been previously analyzed by these techniques (Goday et al., 2006). Using the markers generated by these procedures, the chromosomes of the three species were compared in order to obtain new data that might contribute to a better understanding of the evolution of their karyotypes and of the taxonomic relationships among them. Moreover, the location of ribosomal sequences on the mitotic chromosomes was studied by fluorescence in situ hybridization (FISH).

\section{Material and Methods}

\section{Species}

Anastrepha striata was collected in guavas (Psidium guajava) from the locality of Uberlândia (18 ${ }^{\circ} 51^{\prime} \mathrm{S}, 48^{\circ} 16^{\prime}$ $\mathrm{W})$, State of Minas Gerais; A. bistrigata also from guavas, was collected in Brodowski (2052' S, 47³7' W), State of São Paulo; and A. serpentina from "abricó-da-praia" (Mimusops commersonii) was collected in São Sebastião (2331' S, 4644' W), State of São Paulo. The host fruits were brought to the laboratory and the emerged adults were used to establish colonies maintained in population cages as described by Selivon and Perondini (1998).

\section{Chromosome preparation, staining and in situ hybridization}

Chromosome analyses were performed using third instar larvae collected from fruits routinely used for the maintenance of laboratory colonies. At least 25 larvae of the first generation of each species were used for chromosome preparations. Since the chromosomes of $A$. serpentina were described before by Goday et al. (2006), only a few chromosome preparations were made for the present analysis in order to compare them with the karyotypes of the other two species.

Mitotic chromosome preparations were obtained according to the methods used for Anastrepha species by Goday et al. (2006). Briefly, the imaginal discs and neural ganglia were dissected, transferred to $0.2 \%$ sodium citrate for $13 \mathrm{~min}$, fixed in methanol, acetic acid and water $(11: 11: 2 . \mathrm{v} / \mathrm{v})$ and squashed in $45 \%$ acetic acid. The coverslip was removed after freezing in liquid nitrogen and the preparation was further fixed in $100 \%$ ethanol at $-20{ }^{\circ} \mathrm{C}$, air-dried and stored at $6{ }^{\circ} \mathrm{C}$ in a sealed box with silica gel.

Before staining, the chromosomes were submitted to a denaturing step by incubation in $2 \mathrm{xSSC}$ for $45 \mathrm{~min}$ at $73{ }^{\circ} \mathrm{C}$, washed in ethanol, air-dried, treated for $3 \mathrm{~min}$ in $0.07 \mathrm{~N} \mathrm{NaOH}$, rinsed in ethanol and air-dried.

The slides were immersed in phosphate buffer (PBS), stained with DAPI (4',6-diamidino-2-phenylindole) at a concentration of $0.5 \mu \mathrm{g} / \mathrm{mL}$ in $2 \mathrm{xSSC}$, rinsed in phosphate buffer (PBS) and mounted in PBS+glycerin (1:1) containing the anti-fade n-propyl-gallate. Following the microscopic analysis, the coverslips were removed, the slides washed in PBS and stained with chromomycin A3 at $25 \mu \mathrm{g} / \mathrm{mL}$ in PBS buffer, rinsed in buffer and mounted in PBS+glycerin (1:1) containing the anti-fade n-propylgallate. After recording the images, the coverslips were removed in PBS buffer and the preparations were submitted to C-banding according to Selivon and Perondini (1997).

For detection of ribosomal DNA sequences by FISH, the pDm238 plasmid containing a complete rDNA cistron of Drosophila melanogaster (Tautz et al., 1988) was used as probe and labeled with digoxigenin using the DIG-Nick Translation Mix (Roche Diagnostic GmbH). A $15 \mu \mathrm{L}$ drop of denatured probe was added to the chromosome preparations that had been previously submitted to the denaturing step (as described above), covered with a coverslip and incubated overnight at $37^{\circ} \mathrm{C}$ in a humid chamber. After removal of the coverslip, the slides were thoroughly washed in 2xSSC and in PBS buffer containing 0.1\% Tween 20. The preparations were then covered with a drop of FITCconjugated anti-DIG sheep antibody $(1: 50)$ and left for $30 \mathrm{~min}$ at $37^{\circ} \mathrm{C}$ in a humid chamber. After removal of the coverslip, the slides were washed in $4 \mathrm{xSSC}$ plus $0.01 \%$ Tween 20 at $42{ }^{\circ} \mathrm{C}$, counterstained with DAPI at $0.2 \mu \mathrm{g} / \mathrm{mL}$ in $2 \mathrm{xSSC}$, and mounted in PBS+glycerin (1:1) containing the anti-fade n-propyl-gallate.

\section{Fluorescence microscopy and chromosome measurements}

Chromosome preparations were examined under an Olympus BX60 epifluorescence microscope, using the appropriate filters. Images were digitalized by a Leica DC100 camera coupled to the microscope, and the digital images were pseudocolored (green for DAPI and red for $\mathrm{CMA}_{3}$ staining), merged and edited using the AdobePhotoshop 7.0 software. In the merged pictures four classes of fluorescence were distinguished: bright green, DAPI positive signals; red, $\mathrm{CMA}_{3}$ positive signals; bright yellow, colocalization of $\mathrm{DAPI} / \mathrm{CMA}_{3}$ positive signals, and orange, $\mathrm{CMA}_{3}$ /DAPI positive signals but with preponderance of $\mathrm{CMA}_{3}$ staining.

Chromosome length measurements were performed with the ProImage IPWIN32 software. The length of each chromosome, as well as of the heterochromatic blocks, are expressed as a percentage of the total diploid complement length, according to Robertson (1957). Measurements were performed in five mitoses of each sex, chosen from different individuals.

Solferini and Morgante $(1987,1990)$ numbered the autosomes of Anastrepha species in ascending order, the smallest being number II (or 2), differently from other karyological studies on fruit flies, in which the autosomes were numbered in descending order, number II (or 2) being 
the largest (e.g. Gopalan, 1972; Zacharopoulou, 1986; Procunier and Smith, 1993; Canovai et al., 1996). Considering that the latter identification system is of general use in karyotype analysis, Selivon et al. (2005b) proposed that it should also be adopted in the chromosome analysis of Anastrepha species. Hence, in the present report the autosomes are identified in decreasing order of length, the largest being number II (or 2). Pair I (or 1) is formed by the sex chromosomes.

\section{Results and Discussion}

\section{Anastrepha bistrigata}

In their short description of the chromosomes of this species, Solferini and Morgante (1987) showed that there are two pairs of metacentric and two pairs of acrocentric autosomes, neo-Y/neo-X sex chromosomes, and that the chromosomes $X_{1}$ and $X_{2}$ are acrocentric, while the neo- $Y$ is sub-acrocentric (Figure 1A). The results on the sex chromosomes were corroborated by the present analysis, but autosomes II and IV were shown to have arm ratios (S/L) of 0.70 and 0.72 , respectively, thus being submetacentric instead of metacentric. The size and location of the heterochromatic blocks are shown in Figure 1A and in the idiogram of Figure 3, where the affinity of these blocks to the fluorochromes is also shown. The pericentromeric blocks of autosomes II, III and IV, as well as those of the original $\mathrm{X}_{1}$ and the neo-Y, show colocalization of DAPI/ $\mathrm{CMA}_{3}$-positive signals. Differently, the pericentromeric heterochromatin of autosome $\mathrm{V}$ and the interstitial heterochromatic block of autosome II show a $\mathrm{CMA}_{3} / \mathrm{DAPI}-$ positive signal. In the neo-X and neo-Y chromosomes, the interstitial heterochromatin shows colocalization of DAPI/ $\mathrm{CMA}_{3}$-positive signals, while in autosome IV the block shows a bright DAPI-positive fluorescence.

\section{Anastrepha striata}

According to Solferini and Morgante (1987), the karyotype of this species is composed of two pairs of metacentric and three pairs of acrocentric autosomes with $\mathrm{C}$-bands in their pericentromeric regions. The $\mathrm{X}$ chromosome is acrocentric and the heterochromatic Y chromosome is dot-shaped (Figure 1B). Measurements made in the present analysis showed that the arm ratios of autosomes II
DAPI
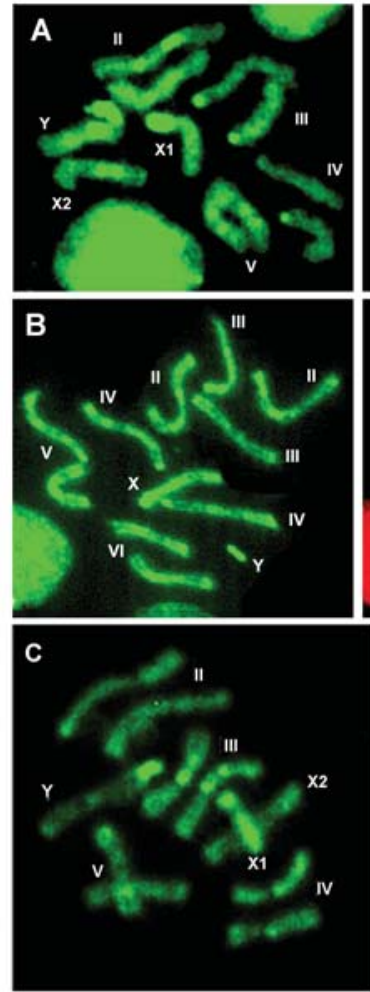

$\mathrm{CMA}_{3}$
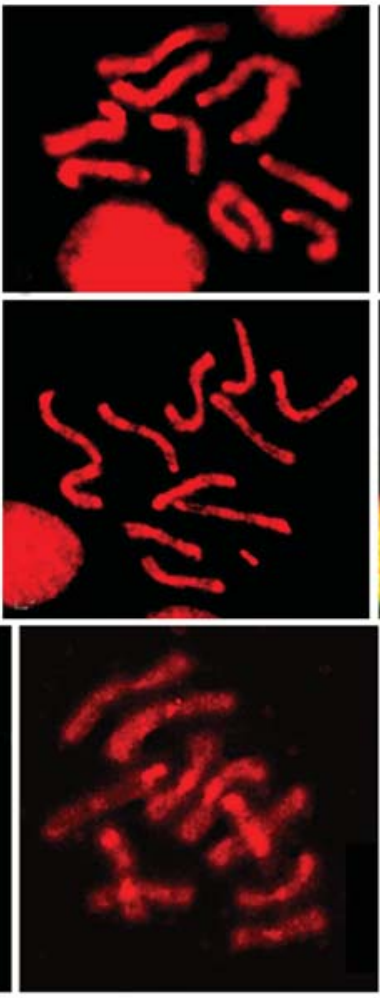

merged
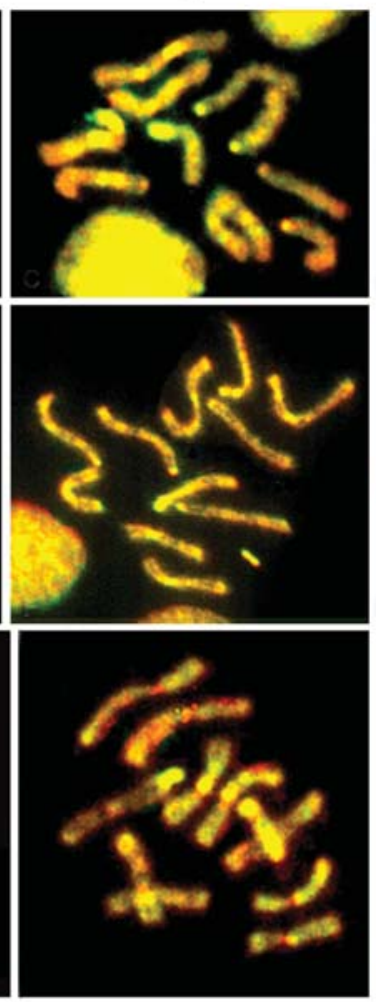

C bands
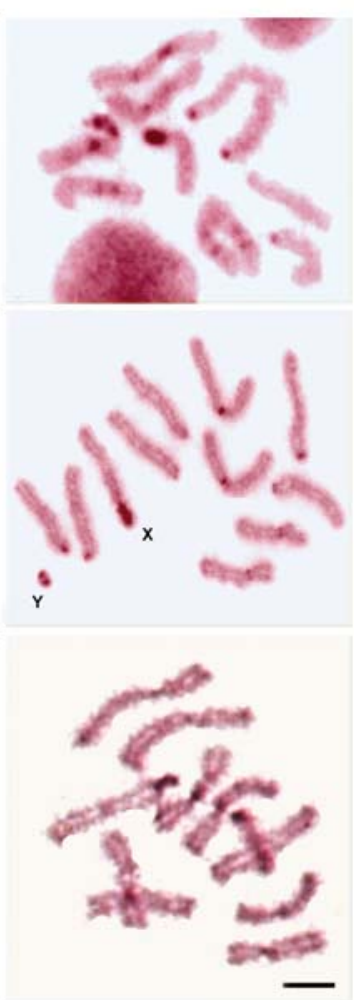

Figure 1 - Metaphases of males of Anastrepha bistrigata, $2 \mathrm{n}=12(\mathrm{~A})$, A. striata, $2 \mathrm{n}=11(\mathrm{~B})$, and A. serpentina, $2 \mathrm{n}=11(\mathrm{C})$, sequentially stained with DAPI and chromomycin A3 followed by C-banding. The original images of the chromosomes stained by the fluorochromes were pseudocolored in green (DAPI) and red $\left(\mathrm{CMA}_{3}\right)$, and the third column shows the merged pictures. The heterochromatic blocks revealed by $\mathrm{C}$-banding showed differential affinity for the fluorochromes, thus indicating that they are either AT- or GC-rich. Only in A. serpentina the pericentromeric heterochromatin of the autosomes is GC-rich. Bright yellow: colocalization of AT- and GC-rich DNA sequences; orange: colocalization of GC- and AT-rich sequences, but with preponderance of GC; green: AT-rich regions; red: GC-rich DNA blocks. Bar represents $10 \mu \mathrm{m}$. 
and $\mathrm{V}$ are, respectively, 0.72 and 0.56 , thus being submetacentric instead of metacentric. The $\mathrm{Y}$ chromosome is a small heterochromatic chromosome. Fluorochrome staining showed colocalization of DAPI/CMA $\mathrm{C}_{3}$-positive signals in the heterochromatic blocks of all chromosomes (Figure 1B). The relative sizes of the chromosomes, as well as of the heterochromatic blocks, are shown in the idiogram of Figure 3.

\section{Anastrepha serpentina}

Solferini and Morgante (1987) described the mitotic chromosomes of this species using C-banding techniques, and recently Goday et al. (2006), using sequential fluorochrome staining, showed that blocks of heterochromatin have differential affinities for the fluorochromes DAPI and $\mathrm{CMA}_{3}$ (Figure 1C). For the comparisons that will be made in the present analysis, the description of the A. serpentina chromosomes will be briefly summarized below and shown in the idiogram in Figure 3. The four autosomes are metacentric and their pericentromeric regions show bright $\mathrm{CMA}_{3}$-positive fluorescence; autosomes II, III and IV possess blocks of heterochromatin showing colocalization of $\mathrm{DAPI} / \mathrm{CMA}_{3}$-positive signals adjacent to the centromeric regions; autosome III has an additional interstitial DAPIpositive heterochromatic block on its long arm; the $\mathrm{X}_{1}$ and $\mathrm{X}_{2}$ chromosomes are acrocentric, and the former has a terminal and an interstitial block of heterochromatin showing colocalization of DAPI/CMA 3 signals; the $\mathrm{X}_{2}$ has no distinguishable heterochromatic region, but usually shows a region with affinity to $\mathrm{CMA}_{3}$ in its proximal tip; the long $\mathrm{Y}$ chromosome has a large block of DAPI-positive heterochromatin at one end, that is divided by a constriction, probably at the centromere location.

\section{Location of ribosomal DNA sequences}

FISH analyses employing an rDNA probe showed hybridization signals only on the sex chromosomes, one site on the $\mathrm{X}$ and one on the $\mathrm{Y}$ chromosome (Figure 2). The
rDNA signals were located in DAPI/CMA 3 -positive heterochromatic blocks at the proximal tips of the $\mathrm{Y}$ and $\mathrm{X}$ chromosomes of $A$. striata, at the proximal tip of the neo-Y and at the posterior border of the heterochromatic block located at the proximal tip of the original X chromosome in $A$. bistrigata. As previously shown by Goday et al. (2006), the signal on the original X chromosome in A. serpentina is located in the interstitial block of the DAPI/CMA $\mathrm{C}_{3}$-positive heterochromatin, while the rDNA on the neo-Y chromosome is located in the DAPI-positive heterochromatic block at the tip of the chromosome.

The presence of a single site of ribosomal genes on both the $\mathrm{X}$ and $\mathrm{Y}$ chromosomes herein observed for $A$. striata and $A$. bistrigata seems to be the usual arrangement in fruit flies, as it has been previously described in eight species of Anastrepha (Goday et al., 2006), in Rhagoletis pomonella (Procunier and Smith, 1993), in Ceratitis capitata (Bedo and Webb, 1989) and in Ceratitis rosa (Wilhoeft and Franz, 1996).

\section{Comparing the karyotypes of the three species}

Comparisons between the karyotypes of $A$. striata and $A$. bistrigata showed substantial similarities with regard to the cytochemical properties of autosomes, as shown in Figure 3. It has been proposed that the karyotype of $A$. bistrigata could have derived from that of A. striata, assuming a fusion of the $\mathrm{Y}$ with autosome III (formerly named V), giving rise to the neo-Y chromosome (Solferini and Morgante, 1990). Figure 3A shows that this hypothesis requires an intermediate step considering that the $\mathrm{X}_{2}$ chromosome and its putative homologous part on the neo-Y chromosome of $A$. bistrigata possess interstitial blocks of heterochromatin which are absent in autosome III of $A$. striata. Since these interstitial blocks show bright DAPI/ $\mathrm{CMA}_{3}$-positive fluorescence and are located at similar positions on these chromosomes, it has to be admitted that an inversion occurred in the autosome of $A$. striata, moving the terminal heterochromatin to an interstitial location, be-

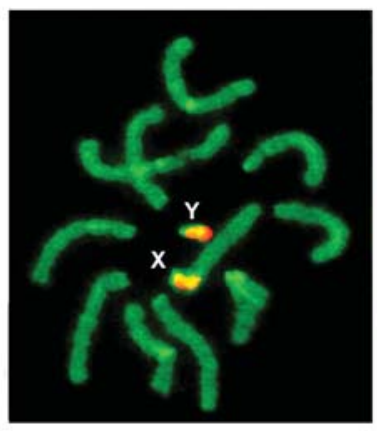

A. striata

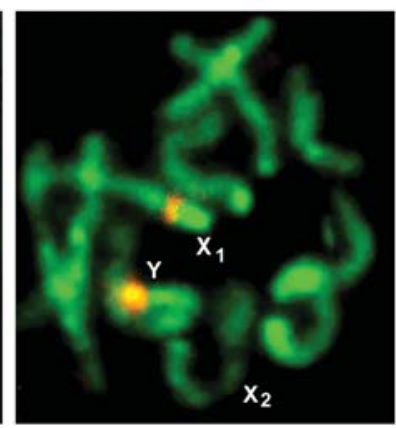

A. bistrigata

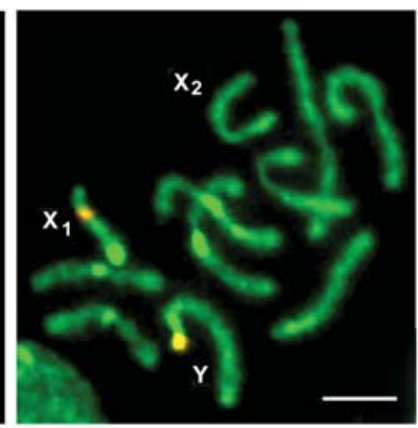

A. serpentina

Figure 2 - Fluorescence in situ hybridization (FISH) of male mitotic chromosomes from the three Anastrepha species with the rDNA pDm238 probe. Merged images of DAPI-stained chromosomes (green) and hybridization signals (red) show that the ribosomal genes are localized in heterochromatic blocks on the sex chromosomes of the three species, one block in the $\mathrm{Y}$ chromosome, and one in the $\mathrm{X}$ of $A$. striata, and in the $\mathrm{X}_{1}$ chromosomes of $A$. bistrigata and $A$. serpentina. Bar represents $10 \mu \mathrm{m}$. 

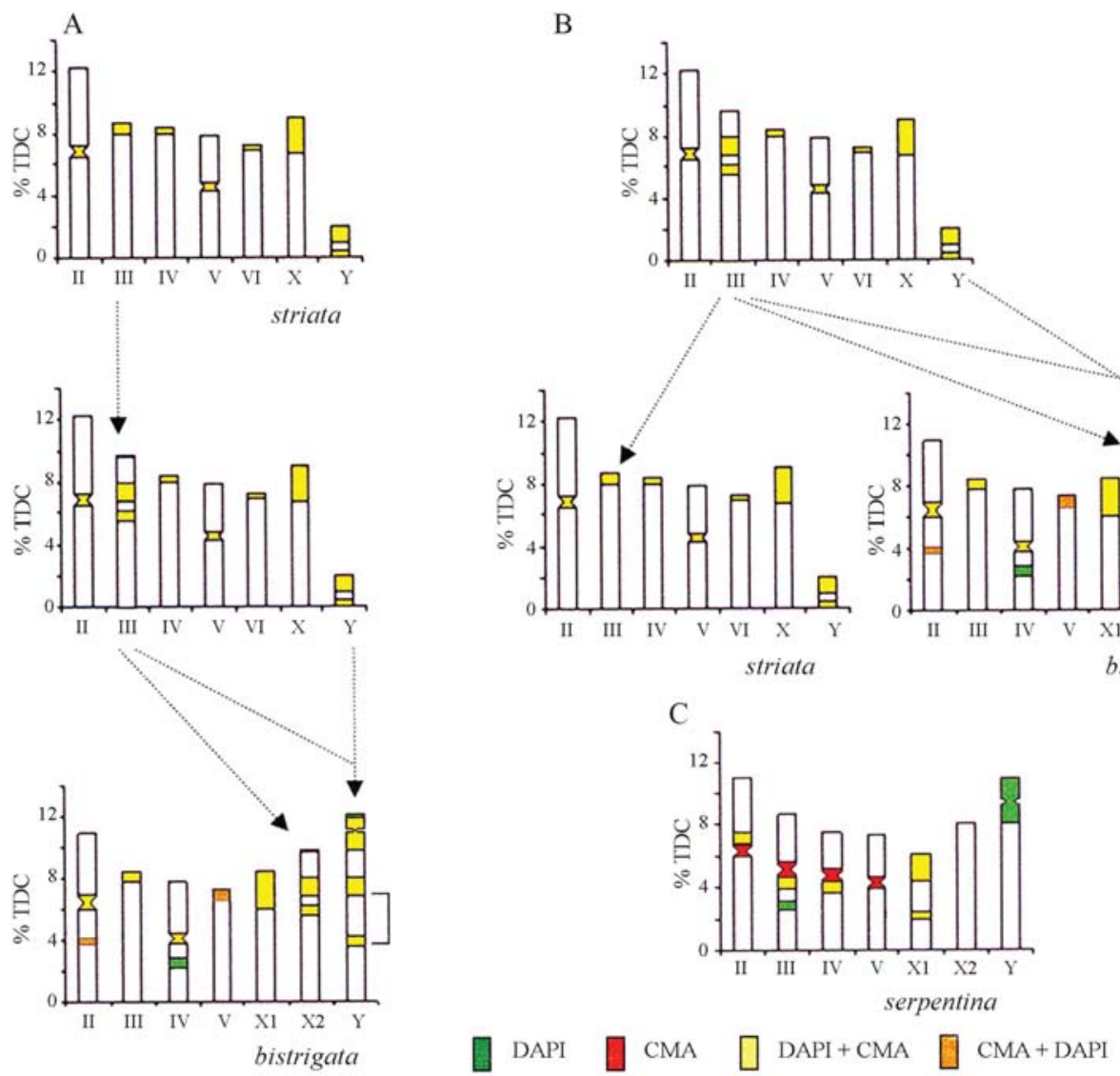

Figure 3 - Idiograms of the mitotic chromosomes of Anastrepha striata, A. bistrigata and A. serpentina, in which the heterochromatic blocks are marked by position, size and affinity for the fluorochromes DAPI and $\mathrm{CMA}_{3}$. Positions of centromeres are indicated by indentation, and in acrocentric chromosomes the centromere is on the top. Color codes as in Figure1. (A) Autosome III of A. striata was proposed as the chromosome involved in the Y:A fusion, originating the neo-X/neo-Y chromosomes of $A$. bistrigata. In the diagram on the left (A), the original karyotype was that of $A$. striata (top diagram), and an inversion in autosome III originated a hypothetical karyotype (middle diagram) from which the A. bistrigata karyotype derived by fusion of autosome III to the Y chromosome (bottom diagram). On the right (B), it is proposed that the karyotypes of A. striata and A bistrigata derived independently from a hypothetical karyotype, considering the same events as in the previous diagram (A). The bracket on the side of the long arm of the $A$. bistrigata neo-Y chromosome indicates the suggested inversion separating the two heterochromatic blocks. In (C), the idiogram of $A$. serpentina shows the greatest differences in affinity of the heterochromatic block to the fluorochromes in relation to the chromosomes of A. bistrigata and A. striata.

fore the fusion of the Y with autosome III. Moreover, a duplication (or a partition) of the heterochromatic block must also have occurred in this new chromosome, since two blocks are present in both the neo-X and the neo-Y chromosomes of $A$. bistrigata. The different locations of these two interstitial heterochromatic blocks in the neo-X compared to the neo-Y chromosome of $A$. bistrigata could be explained by an inversion on either one of these chromosomes, but that would have occurred after the fusion of the $\mathrm{Y}$ with autosome III. An alternative hypothesis (Figure 3B) is that the A. striata and A. bistrigata karyotypes originated from a hypothetical karyotype in which autosome III had two blocks of interstitial heterochromatin. The A. striata karyotype would then have been formed by an inversion and loss of one of the blocks in autosome III, and the $A$. bistrigata karyotype by fusion of the $\mathrm{Y}$ with autosome III which already contained the two blocks of heterochromatin (Figure 3B). The fact that autosomes II and IV of $A$. bistrigata have interstitial heterochromatin that is not present in A. striata autosomes is irrelevant for the hypothesis above, since these blocks could have arisen either before or after the fusion.

Greater differences were observed between the autosomes of $A$. serpentina and the autosomes of the other two species (Figures 1C and 3). The first remarkable difference concerns the pericentromeric regions of the $A$. serpentina autosomes that show bright fluorescence after $\mathrm{CMA}_{3}$ staining, indicating a high content of GC sequences. Actually, these regions appear as DAPI-negative gaps when the chromosomes are stained only with this fluorochrome (Figure 1C). Moreover, the heterochromatin of the neo-Y chromosome shows bright DAPI fluorescence, suggesting that the original $\mathrm{Y}$ chromosome was rich in $\mathrm{AT}$ sequences, differently from the putative original Y of $A$. striata (Figures 1B, 3) and of the neo-Y of $A$. 
bistrigata (Figures 1A, 3), that might have been rich in interspersed $\mathrm{AT}$ and $\mathrm{GC}$ sequences.

\section{Concluding Remarks}

It is difficult to consider a close relationship between the karyotype of $A$. serpentina and those of $A$. striata and $A$. bistrigata. Indeed, it is reasonable to assume, in line with the hypothesis of Solferini and Morgante (1990), that the $A$. serpentina karyotype derived from a hypothetical karyotype with $\mathrm{XY} / \mathrm{XX}$ sex chromosomes and one acrocentric and four metacentric autosomes through fusion of the $\mathrm{Y}$ with the acrocentric autosome, originating the $\mathrm{X}_{1} \mathrm{X}_{2} \mathrm{Y}$ system. These authors also proposed that the karyotype of another species, A. barnesi (leptozona group), that has $\mathrm{XY} / \mathrm{XX}$ sex chromosomes and metacentric autosomes, could be derived from this same hypothetical karyotype, after an inversion transforming the acrocentric autosomes into metacentrics. It is interesting to note that the karyotypes of other Anastrepha species, e.g. A. aphelocentena (mucronota group), A. pseudoparallela (pseudoparallela group), A. spatulata, A. pickeli and A. montei (spatulata group), A. grandis (grandis group) and A. leptozona (leptozona group), also have XY/XX sex chromosomes and metacentric (or submetacentric) autosomes (Bush, 1962; Solferini and Morgante, 1987; Morgante et al., 1996; Selivon et al., 2005b). In contrast, the so far studied species of the more derived fraterculus group, such as A. amita, A. obliqua, A. sororcula, A. turpiniae, A. zenildae and the four cryptic species of $A$. fraterculus, have only acrocentric autosomes (Solferini and Morgante, 1987; Selivon et al., 2004, 2005a, 2005b; Goday et al., 2006). Moreover, it was shown that the centromeric regions of the $A$. grandis autosomes display a bright $\mathrm{CMA}_{3}$ fluorescence, similar to the autosomes of $A$. serpentina (Goday et al., 2006). Thus, the data appear to suggest that the $A$. serpentina karyotype has less affinity to the karyotypes of $A$. bistrigata and $A$. striata than to those of several other Anastrepha species which have metacentric autosomes.

If the serpentina group is monophyletic, as suggested by Norrbom (2002) based on morphological traits, then one would have to postulate that substantial modifications in chromosome structure occurred during the evolution of the group. Alternatively, the present chromosome analysis suggests that the karyotype of $A$. serpentina had an origin which was different from that of the karyotypes of $A$. striata and A. bistrigata. According to this hypothesis, the serpentina species group should be considered as nonmonophyletic or, in agreement with results from phylogenetic analyses based on molecular data (McPheron et al. 1999; Barr et al. 2005), A. bistrigata and A. striata would remain in a group (striata group) while $A$. serpentina would belong to a distinct one (serpentina group), as previously considered in the morphological analysis (Norrbom et al., 1999).

\section{Acknowledgment}

This work was supported by a grant from Fundação de Amparo a Pesquisa do Estado de São Paulo - FAPESP, Brazil (03/02698-3). DS is a fellow of CNPq and FMS had an undergraduate scholarship (IC) from FAPESP (02/09852-5).

\section{References}

Barr NB, Cui L and McPheron BA (2005) Molecular systematics of nuclear gene period in genus Anastrepha (Tephritidae). Ann Entomol Soc Am 98:173-180.

Basso A and Manso F (1998) Are Anastrepha fraterculus chromosomal polymorphism an isolation barrier? Cytobios 93:103-111.

Basso A, Sonvico A, Quesada-Allue LA and Manso F (2003) Karyotypic and molecular identification of laboratory stocks of the South American fruit fly Anastrepha fraterculus (Wied.) (Diptera, Tephritidae). J Econ Entomol 96:12371244.

Bedo DG and Webb GC (1989) Conservation of nucleolar structure in polytene tissues of Ceratitis capitata (Diptera, Tephritidae). Chromosoma 98:443-449.

Bush GL (1962) The cytotaxonomy of the larvae of some Mexican fruit flies in the genus Anastrepha (Tephritidae, Diptera). Psyche 69:87-101.

Canovai R, Macelloni G and Galleni L (1996) Karyology of some Italian populations of the olive fly Bactrocera oleae (Gmelin) (Diptera, Tephritidae). Caryologia 49:247-255.

Goday C, Selivon D, Perondini ALP, Greciano PG and Ruiz MF (2006) Cytological characterization of sex chromosomes and ribosomal DNA location in Anastrepha species (Diptera, Tephritidae). Cytogenet Genome Res 114:70-76.

Gopalan HNB (1972) The study of somatic, meiotic and salivary gland chromosomes of the melonfly, Dacus cucurbitae. Caryologia 25:163-172.

McPheron BA, Han H-Y, Silva JG and Norrbom AL (1999) Phylogeny of the genera Anastrepha and Toxotrypana (Trypetinae, Toxotrypanini) based upon 16S rRNA mitochondrial DNA sequences. In Aluja $\mathrm{M}$ and Norrbom AL (eds) Fruit Flies (Tephritidae): Phylogeny and Evolution of Behaviour. CRC Press, Boca Raton, pp 343-361.

Mendes LOT (1958) Observações citológicas em moscas-dasfrutas. Bragantia 17:29-39

Metz CW (1916) Chromosome studies on the Diptera. II. The paired association of chromosomes in the Diptera, and its significance. J Exp Zool 21:213-279.

Morgante JS, Selivon D, Solferini VN and Matioli SR (1993) Evolutionary patterns in specialist and generalist species of Anastrepha. In: Aluja M and Liedo P (eds) Fruit Flies: Biology and Management. Springer, New York, pp 15-20.

Morgante JS, Selivon D, Solferini VN and Nascimento AS (1996) Genetic and morphological differentiation in the specialist species Anastrepha pickeli and A. monte. In: McPheron BA and Steck GJ (eds) Fruit Flies Pests. St. Lucie Press, Delray Beach, pp 267-272.

Norrbom AL (2002) A revision of the Anastrepha serpentina species group (Diptera, Tephritidae). Proc Entomol Soc Wash 104:390-436. 
Norrbom AL, Zucchi RA and Hernández-Ortiz V (1999) Phylogeny of the genera Anastrepha and Toxotrypana (Trypetinae, Toxotrypanini) based on morphology. In: Aluja M and Norrbom AL (eds) Fruit Flies (Tephritidae): Phylogeny and Evolution of Behaviour. CRC Press, Boca Raton, pp 299342 .

Procunier WS and Smith JJ (1993) Localization of ribosomal DNA in Rhagoletis pomonella (Diptera, Tephritidae) by in situ hybridization. Insect Mol Biol 2:63-174.

Robertson JG (1957) Somatic metaphase chromosomes in geographic isolates of the carrot rust fly, Chamaepsila rosae (F.) (Diptera, Psiliidae). Can J Zool 35:453-458.

Schweizer D (1981) Counterstain-enhanced chromosome banding. Hum Genet 57:1-14.

Selivon D and Perondini ALP (1997) Evaluation of techniques for $\mathrm{C}$ and ASG banding of the mitotic chromosomes of Anastrepha fraterculus (Diptera, Tephritidae). Braz J Genet 20:651-654.

Selivon D and Perondini ALP (1998) Egg-shell morphology in two cryptic species of the Anastrepha fraterculus complex (Diptera, Tephritidae). Ann Entomol Soc Amer 91:473-478.

Selivon D, Perondini ALP and Morgante JS (2005a) A genetic-morphological characterization of two cryptic species of Anastrepha fraterculus complex (Diptera, Tephritidae). Ann Entomol Soc Amer 98:367-381.

Selivon D, Perondini ALP and Rocha LS (2005b) The mitotic chromosomes of eigth species of Anastrepha (Diptera, Tephritidae). Neotrop Entomol 34:273-279.
Selivon D, Vretos C, Fontes L and Perondini ALP (2004) New variant forms in the Anastrepha fraterculus complex (Diptera, Tephritidae). In: Barnes B (ed) Proc. 6th Int Symp Fruit flies of Economic Importance. Isteg Scient. Publ, Irene, pp 253-258.

Solferini VN and Morgante JS (1987) Karyotype study of eight species of Anastrepha (Diptera, Tephritidae). Caryologia 40:229-241.

Solferini VN and Morgante JS (1990) $\mathrm{X}_{1} \mathrm{X}_{1} \mathrm{X}_{2} \mathrm{X}_{2}$ : $\mathrm{X}_{1} \mathrm{X}_{2} \mathrm{Y}$ mechanism of sex determination in Anastrepha bistrigata and $A$. serpentina. Rev Brasil Genet 13:201-208.

Tautz D, Hancock J, Webb D, Tautz C and Dover G (1988) Complete sequences of the rRNA genes of Drosophila melanogaster. Mol Biol Evol 5:287-295.

White I and Elson-Harris MM (1992) Fruit Flies of Economic Significance: Their Identification and Bionomics. CAB International, Wallingford, $599 \mathrm{pp}$.

Wilhoeft U and Franz G (1996) Comparison of the mitotic karyotypes of C. capitata, C. rosa and T. coffea by C-banding and FISH. Genome 39:884-889.

Zacharopoulou A (1986) Cytogenetic analysis of mitotic and salivary gland chromosomes in the Medfly Ceratitis capitata. Genome 29:67-71.

Zucchi RA (2000) Taxonomia. In: Malavasi A and Zucchi R (eds) Moscas-das-Frutas de Interesse Econômico no Brasil: Conhecimento Básico e Aplicado. Holos, Ribeirão Preto, pp 13-24.

Associate Editor: Yatiyo Yonenaga-Yassuda 